\title{
Marine $n-3$ PUFA, heart rate variability and ventricular arrhythmias in patients on chronic dialysis: a cross-sectional study
}

\author{
Jesper M. Rantanen ${ }^{1,2 *}$, Erik B. Schmidt ${ }^{2,3}$, Sam Riahi ${ }^{2,3}$, Søren Lundbye-Christensen ${ }^{3,4}$ and \\ Jeppe H. Christensen ${ }^{1,2}$ \\ ${ }^{1}$ Department of Nephrology, Aalborg University Hospital, Hobrovej 18-22, 9100 Aalborg, Denmark \\ ${ }^{2}$ Department of Clinical Medicine, Aalborg University, Sdr. Skovvej 15, 9000 Aalborg, Denmark \\ ${ }^{3}$ AF Study Group, Department of Cardiology, Aalborg University Hospital, Hobrovej 18-22, 9100 Aalborg, Denmark \\ ${ }^{4}$ Unit of Clinical Biostatistics, Aalborg University Hospital, Sdr. Skovvej 15, 9000 Aalborg, Denmark \\ (Submitted 13 November 2017 - Final revision received 16 February 2018 - Accepted 20 March 2018 - First published online 21 May 2018)
}

\section{Abstract}

Marine $n$-3 PUFA may improve autonomic dysfunction by an increase in heart rate variability (HRV) and may reduce the risk of malignant ventricular arrhythmias. Only a few smaller studies have examined such effects in patients on chronic dialysis, who often have autonomic dysfunction and a high risk of sudden cardiac death, which accounts for almost $30 \%$ of all deaths. This cross-sectional study investigated the association between the plasma phospholipid content of $n$-3 PUFA and 24-h HRV or ventricular arrhythmias in patients on chronic dialysis. A 48-h Holter monitoring was performed on 169 patients on in-centre dialysis (83\%), home haemodialysis (10\%) or peritoneal dialysis (7\%) obtaining data on arrhythmias ( $n$ 152) and 24-h HRV ( $n$ 135). The mean overall HRV (standard deviation of normal intervals (SDNN)) was low and $71 \%$ had a reduced overall HRV (SDNN $<100 \mathrm{~ms})$ indicating autonomic dysfunction. No significant associations between plasma phospholipid content of total marine $n$-3 PUFA, EPA $(22: 5 n-3)$ or DHA $(22: 6 n$-3) and time-domain or frequency-domain HRV were detected in crude or adjusted linear regression analysis. However, a higher plasma phospholipid content of DHA was associated with a significantly lower proportion of patients with ventricular tachycardia (higher DHA-tertile: $9 \% v$. lower DHA-tertile: $28 \%, P=0 \cdot 02$ ). In conclusion, the content of marine $n$-3 PUFA in plasma phospholipids was not associated with 24-h HRV, but a higher plasma phospholipid content of DHA was associated with a lower occurrence of ventricular tachycardia suggesting an antiarrhythmic effect of marine $n-3$ PUFA in patients on chronic dialysis.

Key words: End-stage kidney disease: Heart rate variability: Ventricular arrhythmias: Autonomic dysfunction: $n$-3 PUFA

Patients with end-stage kidney disease (ESKD) have a very high cardiovascular morbidity and mortality compared with the general population. CVD remains the leading cause of death in patients with ESKD, and sudden cardiac death (SCD) is responsible for almost $30 \%$ of all-cause mortality in this population $^{(1,2)}$. SCD is frequently a result of malignant ventricular arrhythmias secondary to abnormalities in ventricular conduction or repolarisation ${ }^{(3)}$. Cardiac autonomic dysfunction is common in patients on chronic dialysis ${ }^{(4)}$ and might be part of the explanation for the high risk of SCD in these patients as an imbalance in the cardiac autonomic modulation may predispose to ventricular arrhythmias ${ }^{(5-7)}$. Heart rate variability (HRV), which is an analysis of beat-to-beat variation of the heart rate (HR), is a non-invasive measure of autonomic modulation of the heart ${ }^{(8)}$. The two most used approaches for the analysis of HRV are time-domain HRV based on simple statistical and geometrical measures of variability and frequency-domain HRV from power spectral analysis. Autonomic dysfunction characterised by excessive sympathetic activity and/or inadequate parasympathetic tone can be demonstrated by an attenuated $\operatorname{HRV}^{(7,8)}$, which has been shown to be a strong independent predictor of mortality and SCD especially in patients after a myocardial infarction ${ }^{(5,6,8)}$, but also in other populations including patients on chronic dialysis ${ }^{(7,9-12)}$.

The marine $n-3$ PUFA, DHA (22:6n-3) and EPA (20:5n-3) are essential fatty acids mainly derived from fatty fish, and the intake of these fatty acids can be assessed by measurements of content in plasma phospholipids ${ }^{(13)}$. Marine $n-3$ PUFA have several cardiovascular benefits and have been associated with a reduction in cardiovascular mortality and SCD in some studies ${ }^{(14-17)}$ suggesting an antiarrhythmic effect, which also has been seen in animal and in vitro studies ${ }^{(18)}$. The possible antiarrhythmic mechanisms are not clear, but may include a direct effect on the myocardium by incorporation $n-3$ PUFA in

Abbreviations: HD, haemodialysis; HF, high frequency; HRV, heart rate variability; LF, low frequency; PD, peritoneal dialysis; SCD, sudden cardiac death; SDNN, standard deviation of normal intervals; VT, ventricular tachycardia.

* Corresponding author: J. M. Rantanen, fax +459766 3806, email jemr@rn.dk 
the bilayer of the cardiomyocyte cell membrane changing the cellmembrane fluidity or directly affecting ion channels in the cardiomyocytes $^{(18,19)}$, but also an indirect effect by changing autonomic modulation of the heart shown by an increase in $\operatorname{HRV}^{(18,20)}$.

Associations between marine $n$-3 PUFA, HRV and arrhythmias have only been addressed in a few smaller studies in chronic dialysis patients ${ }^{(21,22)}$. Hence, the aim of this study was to examine the possible associations between plasma phospholipid content of total marine $n-3$ PUFA, EPA or DHA and 24-h HRV or ventricular arrhythmias in patients on chronic dialysis.

\section{Methods \\ Setting and participants}

In a cross-sectional design, chronic dialysis patients from Aalborg University Hospital and its satellite dialysis units treating all patients in the northern part of Denmark were recruited from October 2013 to November 2015. Inclusion criteria were treatment with haemodialysis (HD), home HD or peritoneal dialysis (PD) for $\geq 3$ months and age $>18$ years. The only exclusion criterion was the inability to give informed consent. The study protocol was approved by the North Denmark Region Committee on Health Research Ethics and all participants provided written informed consent.

\section{Measurements}

In-centre dialysis patients were examined at the dialysis unit. Home HD patients were invited to have a standard HD session at the dialysis unit on the day of the examination, but if they declined ( $n$ 9) they were examined in the outpatients clinic and received dialysis at home as usual. Patients treated with PD followed their normal individual schedule of treatment. At the day of the examination, the following data were obtained in each patient: detailed medical history, anthropometric data, blood pressure measurements (the mean of the last two of three readings after at least $5 \mathrm{~min}$ rest), venous blood samples, and a 48-h Holter monitoring. Regarding in-centre patients, the Holter monitoring started just before the initiation of a dialysis session and thus day 1 represented a dialysis day and day 2 a non-dialysis day. Patients were encouraged to maintain normal daily activities and abstain from strenuous exercise during the recording.

\section{Laboratory methods}

Blood samples were drawn non-fasting for practical reasons in the morning or the afternoon following the patients' normal dialysis schedule. Pre-HD blood samples were obtained from the HD access according to the usual procedure at the department in in-centre patients and from venepuncture in patients examined in the outpatient clinic.

Routine haematologic and biochemical analysis were performed according to standard routines in the central hospital laboratory. Blood collected for plasma phospholipid fatty acid analysis were flushed with liquid $\mathrm{N}_{2}$ to avoid oxidation and stored at $-80^{\circ} \mathrm{C}$ until later analysis at the Lipid Research Center, Aalborg University Hospital. The analysis was done as previously described $^{(23)}$, but in short, the fatty acid composition in the phospholipid fraction was measured by GC (Varian 3900; Varian) and the results expressed as weight percent (wt\%) of total fatty acids. Total marine $n$-3 PUFA were defined as the sum of DHA, EPA and DPA.

\section{Holter monitoring and heart rate variability analysis}

A 48-h ECG recording was obtained in all patients using a four-lead two-channel digital recorder (Lifecard CF; Del Mar Reynolds Medical Limited). By using Pathfinder Digital 700 (Reynolds Medical Limited) initial editing of the recording was done using an interactive method with visual confirmation of all arrhythmias, premature atrial complexes, and premature ventricular complexes (PVC). All non-sinus beats and artifacts were excluded from the HRV analysis, which was done for days 1 and 2 separately. HRV analysis was only considered valid and used in the final analysis if at least $1152 \mathrm{~min}(80 \%$ of $24 \mathrm{~h}$ ) of the recording were analysable with sinus beats. The time-domain HRV analyses were also done using Pathfinder Digital 700 (Reynolds Medical Limited). The following standard timedomain HRV variables were analysed: the mean of all normal RR-intervals (mean RR-interval); the standard deviation of all normal RR-intervals (SDNN); the mean of the standard deviation of all normal RR-intervals for all 5-min segments (SDNNi); the standard deviation of the mean of RR-intervals in successive 5-min segments (SDANN); the square root of the mean of the sum of the squares of differences between adjacent intervals (rMSSD); and the total number of all RR-intervals divided by the height of the histogram of all RR-intervals measured in a discrete scale with bins of $7 \cdot 8125 \mathrm{~ms}$ (triangular index). The edited files were exported to HRV tools (Reynolds Medical Limited) to perform power spectral analysis using Fast Fourier Transformation for the following frequency-domain measures: low frequency (LF, 0.04-0.15 Hz), high frequency (HF, 0.15-0.4 Hz) and LF:HF ratio. LF and HF were also given as normalised units, which reflects the relative value of each power component in proportion to total power minus very-low-frequency component. The frequency-domain analyses were done in 5-min segments with at least $90 \%$ of each segment consisting of normal RR-intervals and then averaged on the entire 24-h recording. The following ventricular arrhythmias were evaluated: the number of PVC, the occurrence of frequent PVC ( $\geq 30 / \mathrm{h}$ ) and occurrence of ventricular tachycardia (VT) defined as $\geq 3$ ventricular beats with a $\mathrm{HR}>100$ beats per min.

\section{Statistical analysis}

All statistical analyses were performed using Stata 14 (2015; StataCorp) and a two-tailed $P$ value of $<0.05$ was considered statistically significant. Continuous variables were expressed as means and standard deviation or medians with interquartile ranges for normal distributed or skewed data, respectively. Categorical variables were expressed as frequencies and percentages. Comparisons of HRV between days 1 and 2 were done by paired $t$ test or Wilcoxon's signed-rank test depending on the distribution for patients with available data on both days. Comparisons of HRV between groups were made using Student's $t$ test or one-way ANOVA for normally distributed data and 
Wilcoxon's rank sum test or Kruskal-Wallis test for skewed data. Simple linear regression was used to analyse relationships between plasma phospholipid levels of $n-3$ PUFA and HRV while multivariate linear regression was done to control for confounders. Using an epidemiological approach, it was a priori decided to adjust for the following covariates: demography (age; sex; smoking status), co-morbidity (diabetes mellitus; CHD) and the use of medication (beta blocker; angiotensin converting enzyme-inhibitor or angiotensin receptor blocker; statin). Sensitivity analysis was done by bootstrapping with 1000 replications to estimate standard errors and calculate estimated CI. Furthermore, the analyses were repeated excluding the most evident outliers. Regarding ventricular arrhythmias, these were analysed according to groups of $n-3$ PUFA tertiles. The $\chi^{2}$ test was applied for comparison of categorical variables. Multiple logistic regression were performed to adjust for confounding, but due to a low event count adjustments were only done for age, sex and CHD to prevent overfitting of the model.

\section{Results}

\section{Patient characteristics}

A total of 169 patients were enrolled (Fig. 1). Baseline characteristics for the total population and patients with a least $1 \mathrm{~d}$ recording analysed for HRV ( $n$ 135) are listed in Table 1 . The treatment modalities were in-centre dialysis (83\%), home HD (10\%) and PD (7\%). The causes of ESKD were diabetes mellitus (28\%) glomerulonephritis (18\%); obstructive nephropathy, reflux or chronic pyelonephritis (13\%); polycystic kidney disease (10\%); hypertension and ischaemic nephropathy (9\%) or other known and unknown causes (22\%).

\section{Heart rate variability on days 1 and 2}

HRV analysis was possible in 129 and 122 patients on days 1 and 2, respectively. We only obtained HRV on both days in 116 patients (Table 2). The patients had a low overall 24-h HRV and assessed by the highest measured SDNN on days 1 or 2 , $29 \%$ had a normal HRV (SDNN>100 ms), $61 \%$ had a moderately reduced HRV (SDNN 50-100 ms) and 10\% had a severely reduced HRV $(\mathrm{SDNN}<50 \mathrm{~ms})^{(8)}$. The mean RR-interval and the SDNNi were higher on day 1 compared with days 2 , but there were no other statistically significant differences between the $2 \mathrm{~d}$ concerning other time-domain indices. Regarding frequency-domain HRV, we observed a significantly higher LF, normalised LF and LF:HF ratio and a lower normalised HF on day 1 compared with day 2 . To clarify the effect of the dialysis session on HRV a subgroup analysis was done including patients treated with in-centre dialysis and only having HD on day 1 ( $n$ 92). This analysis showed similar results (data not shown). CHD and diabetes mellitus were both associated with a lower SDNN, whereas no differences were seen between men and women or between dialysis modalities (Fig. 2).

\section{n-3 PUFA and heart rate variability}

No significant associations were observed between the contents of DHA or EPA in plasma phospholipids and time-domain or

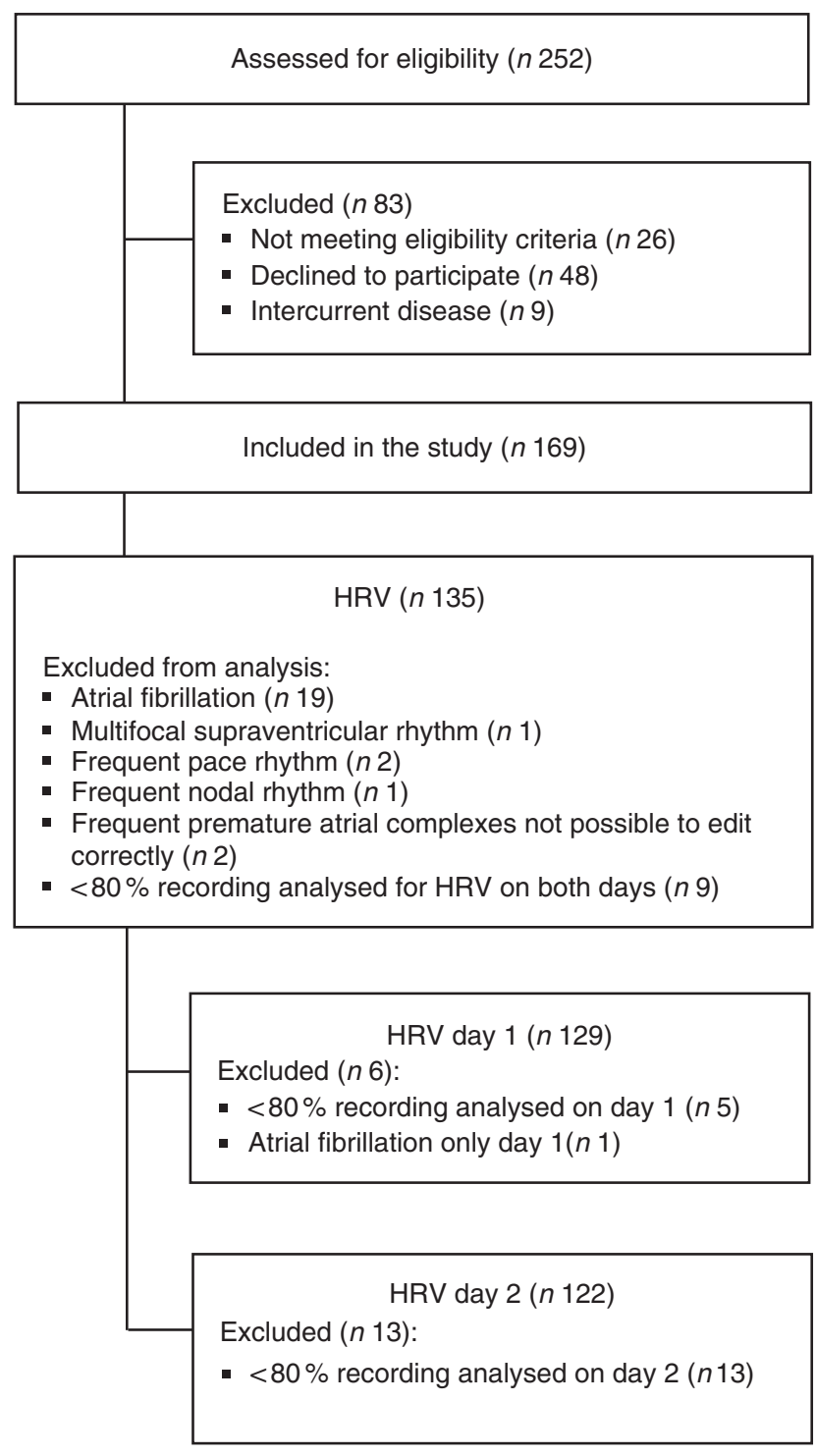

Fig. 1. Study flow chart. HRV, heart rate variability.

frequency-domain HRV in the crude or adjusted multiple linear regression analysis (Fig. 3 and Table 3). Analyses were also done for total marine $n$ - 3 PUFA with the similar results of no association with HRV (data not shown). Sensitivity analysis using bootstrap methods showed similar results (data not shown). A few patients had very high levels of the exposure variable ( $n-3$ PUFA) and repeating the analyses excluding these outliers did not change the results. For the main overall 24-h HRV parameter SDNN mostly used for risk stratification for SCD, we performed additional subanalyses with data stratified by modality, sex, the presence of diabetes mellitus and presence of CHD. These subanalyses also showed no significant associations between DHA, EPA or total marine $n$ - 3 PUFA and SDNN (data not shown).

\section{n-3 PUFA and ventricular arrhythmias}

Arrhythmias analyses on recordings with $>80 \%$ of the 24-h was obtainable in 152 patients. Patients were grouped according to 
Table 1. Baseline characteristics for all patients and patients with heart rate variability (HRV) analysis

(Percentages and numbers; mean values and standard deviations; medians and interquartile ranges (IQR))

\begin{tabular}{|c|c|c|c|c|}
\hline & \multicolumn{2}{|c|}{$\begin{array}{l}\text { All patients } \\
(n 169)\end{array}$} & \multicolumn{2}{|c|}{$\begin{array}{l}\text { HRV analysis } \\
\quad(n \text { 135) }\end{array}$} \\
\hline & $n$ & $\%$ & $n$ & $\%$ \\
\hline \multicolumn{5}{|l|}{ Sex } \\
\hline Male & 110 & $65 \cdot 1$ & 86 & 63.7 \\
\hline Female & 59 & 34.9 & 49 & $36 \cdot 3$ \\
\hline \multicolumn{5}{|l|}{ Ethnicity } \\
\hline Caucasian & 165 & 97.6 & 132 & 97.8 \\
\hline Asian & 4 & $2 \cdot 4$ & 3 & $2 \cdot 2$ \\
\hline Current smoking & 34 & $20 \cdot 1$ & 29 & 21.5 \\
\hline \multicolumn{5}{|l|}{ Co-morbidity } \\
\hline Hypertension & 153 & 90.5 & 124 & 91.9 \\
\hline Diabetes mellitus, type 1 & 21 & $12 \cdot 4$ & 21 & $15 \cdot 6$ \\
\hline Diabetes mellitus, type 2 & 39 & $23 \cdot 1$ & 29 & 21.5 \\
\hline $\mathrm{CHD}$ & 52 & 30.8 & 37 & $27 \cdot 4$ \\
\hline Ischaemic stroke & 35 & $20 \cdot 7$ & 26 & $19 \cdot 3$ \\
\hline Haemorrhagic stroke & 10 & $5 \cdot 9$ & 8 & 5.9 \\
\hline Peripheral artery disease & 32 & 18.9 & 20 & $14 \cdot 8$ \\
\hline \multicolumn{5}{|l|}{ Medication } \\
\hline Beta blocker & 107 & 63.3 & 83 & 61.5 \\
\hline ACE inhibitor & 32 & 18.9 & 26 & $19 \cdot 3$ \\
\hline ARB & 45 & $26 \cdot 6$ & 39 & 28.9 \\
\hline \multirow[t]{2}{*}{ Statin } & 65 & 38.5 & 54 & $40 \cdot 0$ \\
\hline & Mean & SD & Mean & SD \\
\hline Age (years) & $62 \cdot 3$ & $14 \cdot 1$ & 59.9 & $14 \cdot 2$ \\
\hline \multicolumn{5}{|l|}{ Dialysis vintage (years) } \\
\hline Median & \multirow{2}{*}{\multicolumn{2}{|c|}{$\begin{array}{c}1.80 \\
0.62,5 \cdot 67\end{array}$}} & \multicolumn{2}{|c|}{1.70} \\
\hline IQR & & & 0.59 & 5.46 \\
\hline Weight $(\mathrm{kg})^{*}$ & 78.8 & $18 \cdot 7$ & $78 \cdot 6$ & $19 \cdot 0$ \\
\hline BMI $\left(\mathrm{kg} / \mathrm{m}^{2}\right)$ & $27 \cdot 1$ & $6 \cdot 2$ & $27 \cdot 0$ & $6 \cdot 1$ \\
\hline \multicolumn{5}{|l|}{ Blood pressure $(\mathrm{mmHg}) \dagger$} \\
\hline Systolic & 143.3 & $25 \cdot 9$ & $145 \cdot 3$ & 24.3 \\
\hline Diastolic & 73.0 & $13 \cdot 1$ & $75 \cdot 0$ & 12.5 \\
\hline \multicolumn{5}{|l|}{ Blood } \\
\hline $\mathrm{Hb}(\mathrm{mmol} / \mathrm{l})$ & $7 \cdot 0$ & 0.8 & $7 \cdot 0$ & 0.9 \\
\hline \multicolumn{5}{|l|}{ Plasma } \\
\hline \multicolumn{5}{|l|}{ C-reactive protein $(\mathrm{mg} / \mathrm{l})$} \\
\hline Median & \multirow{2}{*}{\multicolumn{2}{|c|}{$\begin{array}{c}3.6 \\
1.9,9.6\end{array}$}} & \multirow{2}{*}{\multicolumn{2}{|c|}{$\begin{array}{c}3.3 \\
1.6 .9 .0\end{array}$}} \\
\hline IQR & & & & \\
\hline Albumin (g/l) & $32 \cdot 2$ & 3.9 & $32 \cdot 3$ & 3.9 \\
\hline \multicolumn{5}{|l|}{ High-sensitive troponin $\mathrm{T}(\mathrm{ng} / \mathrm{l})$} \\
\hline Median & \multirow{2}{*}{\multicolumn{2}{|c|}{$\begin{array}{c}57 \\
33,98\end{array}$}} & \multirow{2}{*}{\multicolumn{2}{|c|}{$\begin{array}{c}53 \\
32 \quad 94\end{array}$}} \\
\hline IQR & & & & \\
\hline Total cholesterol $(\mathrm{mmol} / \mathrm{l})$ & 4.3 & 1.4 & 4.4 & 1.4 \\
\hline HDL-cholesterol (mmol/l) & $1 \cdot 19$ & 0.40 & $1 \cdot 19$ & 0.41 \\
\hline LDL-cholesterol (mmol/l) & 2.35 & $1 \cdot 16$ & $2 \cdot 38$ & 1.20 \\
\hline \multicolumn{5}{|l|}{ TAG (mmol/l) } \\
\hline Median & \multirow{2}{*}{\multicolumn{2}{|c|}{$\begin{array}{c}1.5 \\
1.0 .2 .2\end{array}$}} & 1 & \\
\hline IQR & & & 1.0, & $2 \cdot 3$ \\
\hline Plasma phospholipid (weight \%) & & & & \\
\hline $20: 5 n-3$ (EPA) & 1.69 & $1 \cdot 18$ & 1.69 & 1.24 \\
\hline $22: 6 n-3(\mathrm{DHA})$ & 4.17 & $1 \cdot 24$ & $4 \cdot 15$ & 1.25 \\
\hline Total $n-3$ PUFA (EPA + DHA + DPA) & 6.97 & $2 \cdot 25$ & 6.95 & $2 \cdot 31$ \\
\hline
\end{tabular}

$\mathrm{ACE}$, angiotensin converting enzyme; $\mathrm{ARB}$, angiotensin receptor blocker.

* Weight: measured after ultrafiltration in in-centre patients.

† Measurement before haemodialysis on in-centre patients, and at a random time in patients examined in the outpatient clinic.

marine $n-3$ PUFA tertiles to assess the associations of $n$ - 3 PUFA and ventricular arrhythmias (Table 4). There were no significant associations with ventricular arrhythmias regarding total marine $n-3$ PUFA or EPA. However, the content of DHA in plasma phospholipids was associated with a significantly lower proportion of patients with non-sustained VT (Fig. 4). Comparing the lower and upper tertiles of DHA the odds for the presence of VT was 3.8-fold $(P=0.02)$ and 6.9-fold $(P=0.002)$ higher in the lowest tertile of DHA in crude and adjusted logistic regression analysis, respectively.

\section{Discussion}

The present study is to our knowledge the largest study investigating the associations between $n-3$ PUFA and HRV in patients on chronic dialysis. This is also the first study to address associations between ventricular arrhythmias documented by Holter monitoring with plasma content of $n-3$ PUFA in patients on chronic dialysis. We were unable to demonstrate any associations between $n$-3 PUFA levels in plasma phospholipids and HRV. However, higher plasma phospholipid levels of DHA were associated with a lower proportion of patients with nonsustained VT.

The patients in our study had an attenuated 24-h overall HRV (SDNN) at a similar or even lower level than most earlier studies in patients on chronic dialysis ${ }^{(4,21,24)}$. Furthermore, $10 \%$ had a severely reduced overall HRV (SDNN $<50 \mathrm{~ms}$ ) which may be explained by a population with a high level of co-morbidities including CHD and diabetes mellitus, which often lead to a more pronounced reduction in $\mathrm{HRV}^{(4,8,9)}$, which we also observed in our study. Moreover, we did not observe any difference of overall HRV (SDNN and triangular index) between day 1 (the dialysis day for in-centre patients) and day 2 (without dialysis), but we did see a significantly higher LF, normalised LF and LF:HF ratio and a lower normalised HF on day 1 compared with day 2. This may be interpreted as a change in the sympatho-vagal balance toward a decrease in vagal modulation and/or an increase in sympathetic modulation on the dialysis day compared with the non-dialysis day, which may be explained by the cardiovascular stress caused by ultrafiltration-induced intravascular hypovolemia on the dialysis day ${ }^{(25)}$.

Evidence of associations between intake of fish or $n-3$ PUFA and the cardiovascular outcome is sparse in patients on chronic dialysis. In a retrospective study, Kutner et al. ${ }^{(26)}$ reported that fish intake was associated with reduced mortality in 216 incident PD and HD patients. In some epidemiologic studies $\mathrm{DHA}^{(27,28)}, \mathrm{EPA}^{(29)}$ and the Omega-3 index (EPA+DHA in erythrocytes $)^{(30)}$ have also been associated with significantly lower mortality in HD patients. In a case-control study ${ }^{(31)}$ of 100 HD patients who died of SCD and 300 patients, who survived the 1st year of dialysis, a striking inverse relationship between serum phospholipid total marine $n$ - 3 PUFA and the odds of SCD was observed. In contrast to this, Huang et al. ${ }^{(32)}$ reported that plasma levels of linoleic acid, but not of DHA, EPA or DPA was associated with lower mortality in 222 incident PD and HD patients before kidney transplantation. Only two randomized controlled trials have assessed n-3 PUFA supplementation on cardiovascular events in patients on chronic dialysis. A study from our institution ${ }^{(33)}$ showed no effect of supplementation of $1.7 \mathrm{~g}$ marine $n$-3 PUFA for 2 years on a composite primary endpoint of total cardiovascular events and 
Table 2. Heart rate variability on 2 consecutive days in patients on chronic dialysis (Mean values and standard deviation; medians and interquartile ranges (IQR))

\begin{tabular}{|c|c|c|c|c|c|c|c|c|c|}
\hline & \multicolumn{4}{|c|}{ Day $1^{*}$} & \multicolumn{4}{|c|}{ Day $2^{*}$} & \multirow[b]{3}{*}{$P \dagger$} \\
\hline & \multicolumn{2}{|c|}{ All $(n$ 129) } & \multicolumn{2}{|c|}{ Paired $(n 116)$} & \multicolumn{2}{|c|}{ All $(n$ 122) } & \multicolumn{2}{|c|}{ Paired $(n 116)$} & \\
\hline & Mean & SD & Mean & SD & Mean & SD & Mean & SD & \\
\hline \multicolumn{10}{|l|}{ Time-domain } \\
\hline Mean RR (ms) & 819.7 & $120 \cdot 3$ & 818.1 & 121.0 & $803 \cdot 2$ & $115 \cdot 0$ & 803.0 & $115 \cdot 8$ & $<0.001$ \\
\hline SDNN (ms) & $83 \cdot 8$ & 33.3 & $83 \cdot 6$ & $32 \cdot 8$ & 81.8 & 34.0 & $83 \cdot 1$ & 34.3 & 0.76 \\
\hline \multicolumn{10}{|l|}{ SDNNi (ms) } \\
\hline Median & \multirow{2}{*}{\multicolumn{2}{|c|}{$\begin{array}{c}26 \\
19,36\end{array}$}} & \multirow{2}{*}{\multicolumn{2}{|c|}{$\begin{array}{c}27 \\
19,36\end{array}$}} & \multirow{2}{*}{\multicolumn{2}{|c|}{$\begin{array}{c}24 \\
18,34\end{array}$}} & \multirow{2}{*}{\multicolumn{2}{|c|}{$\begin{array}{c}25 \\
18,36\end{array}$}} & 0.02 \\
\hline IQR & & & & & & & & & \\
\hline \multicolumn{10}{|l|}{ SDANN (ms) } \\
\hline Median & \multirow{2}{*}{\multicolumn{2}{|c|}{$\begin{array}{c}70 \\
55,92\end{array}$}} & \multirow{2}{*}{\multicolumn{2}{|c|}{$\begin{array}{c}71 \\
56,93\end{array}$}} & \multirow{2}{*}{\multicolumn{2}{|c|}{$\begin{array}{c}69 \\
52,88\end{array}$}} & \multirow{2}{*}{\multicolumn{2}{|c|}{$\begin{array}{c}70 \\
54,90\end{array}$}} & 0.96 \\
\hline \multicolumn{2}{|l|}{ rMSSD(ms) } & & & & & & & & \\
\hline Median & \multirow{2}{*}{\multicolumn{2}{|c|}{$\begin{array}{c}13 \\
9,17\end{array}$}} & \multirow{2}{*}{\multicolumn{2}{|c|}{$\begin{array}{c}12 \\
9,17\end{array}$}} & \multicolumn{2}{|c|}{12} & \multirow{2}{*}{\multicolumn{2}{|c|}{$\begin{array}{c}12 \\
9,16\end{array}$}} & $0 \cdot 13$ \\
\hline IQR & & & & & & & & & \\
\hline \multicolumn{10}{|l|}{ Triangular Index } \\
\hline Median & \multicolumn{2}{|c|}{21} & \multicolumn{2}{|c|}{21} & \multicolumn{2}{|c|}{20} & \multirow{2}{*}{\multicolumn{2}{|c|}{$\begin{array}{c}21 \\
15,27\end{array}$}} & 0.62 \\
\hline IQR & \multicolumn{2}{|c|}{16,27} & \multicolumn{2}{|c|}{16,27} & \multicolumn{2}{|c|}{15,27} & & & \\
\hline $\begin{array}{l}\text { Frequency-domain } \\
\qquad \operatorname{LF}\left(\mathrm{ms}^{2}\right)\end{array}$ & & & & & & & & & \\
\hline Median & & & & & & & & & 0.007 \\
\hline IQR & & & & & & & & & \\
\hline LF (nu) & $61 \cdot 1$ & $19 \cdot 7$ & $63 \cdot 1$ & 18.5 & $60 \cdot 0$ & $19 \cdot 7$ & $60 \cdot 8$ & $19 \cdot 1$ & 0.001 \\
\hline $\mathrm{HF}\left(\mathrm{ms}^{2}\right)$ & & & & & & & & & \\
\hline Median & & & & & & & & & 0.63 \\
\hline IQR & & & & & & & & & \\
\hline $\begin{array}{l}\text { HF }(n u) \\
\text { LF:HF ratio }\end{array}$ & $28 \cdot 3$ & 13.7 & $27 \cdot 2$ & $12 \cdot 6$ & $29 \cdot 3$ & $14 \cdot 6$ & 28.9 & $14 \cdot 3$ & 0.01 \\
\hline Median & & & & & & & & & 0.02 \\
\hline IQR & 1.3 & & & & 1.4 & 93 & & & \\
\hline
\end{tabular}

Mean RR, 24-h mean value of RR-intervals; SDNN, 24-h standard deviation of normal intervals; SDANN, standard deviation of the mean of RR-intervals in successive 5-min segments; SDNNi, mean of the standard deviation of all normal RR-intervals for all 5-min segments; rMSSD, square root of the mean of the sum of the squares of differences between adjacent intervals; LF, low frequency; nu, normalised units; HF, high frequency.

* Day 1 was a dialysis day and day 2 a non-dialysis day in patients on in-centre dialysis.

† Comparisons between days were made on paired data using either paired $t$ test or signed-rank test.
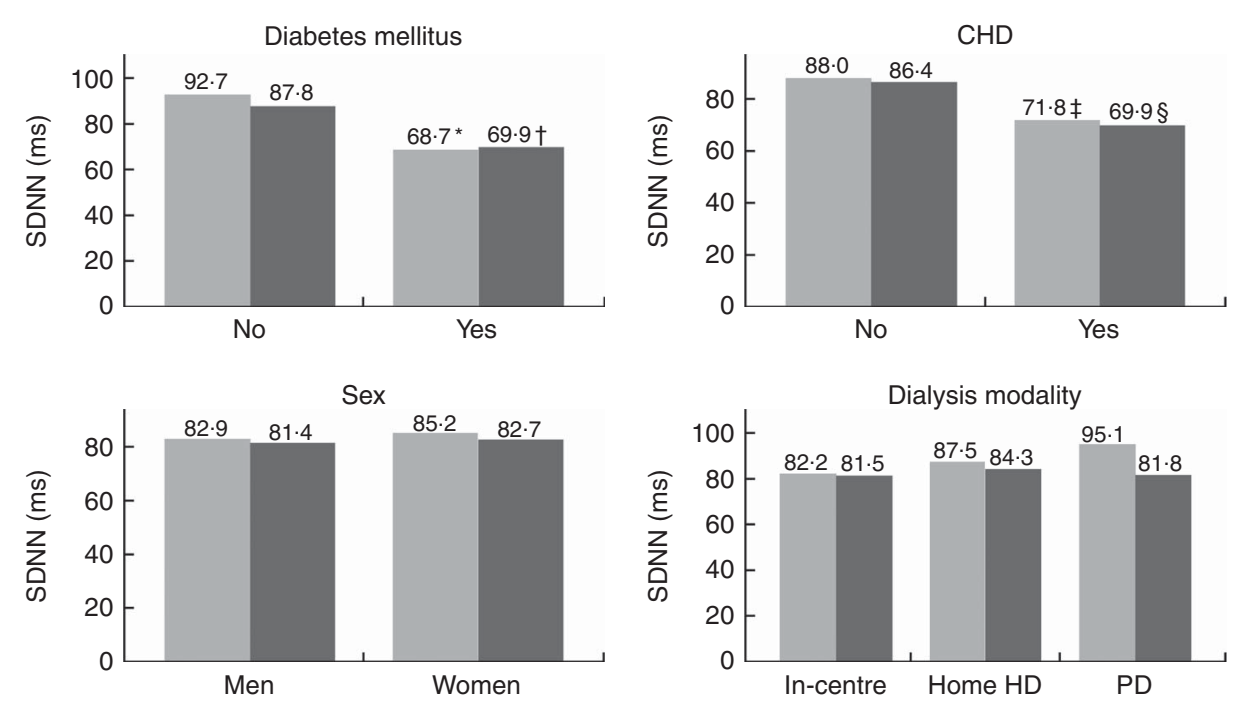

Fig. 2. Mean 24-h standard deviation of normal intervals (SDNN) stratified by DM, CHD, sex or dialysis modality. Significantly lower SDNN was observed with the presence of $\mathrm{DM}\left({ }^{*} P=0.0001 ; \dagger P=0.005\right)$ or $\mathrm{CHD}(\ddagger P=0.01$ and $\S P=0.02)$. Comparisons between groups were made for each day. HD, haemodialysis; PD, peritoneal dialysis; 1 , day $1 ;$, day 2 .

death in 206 HD patients with known CHD, but a significant reduction in the risk of myocardial infarction as a secondary outcome was observed. In another study on arterio-venous-graft patency Lok et $a l^{(34)}$ demonstrated (as a secondary outcome) an improved cardiovascular event-free survival in patients treated with $2 \cdot 4 \mathrm{~g} n$-3 PUFA compared with placebo for 1 year. 

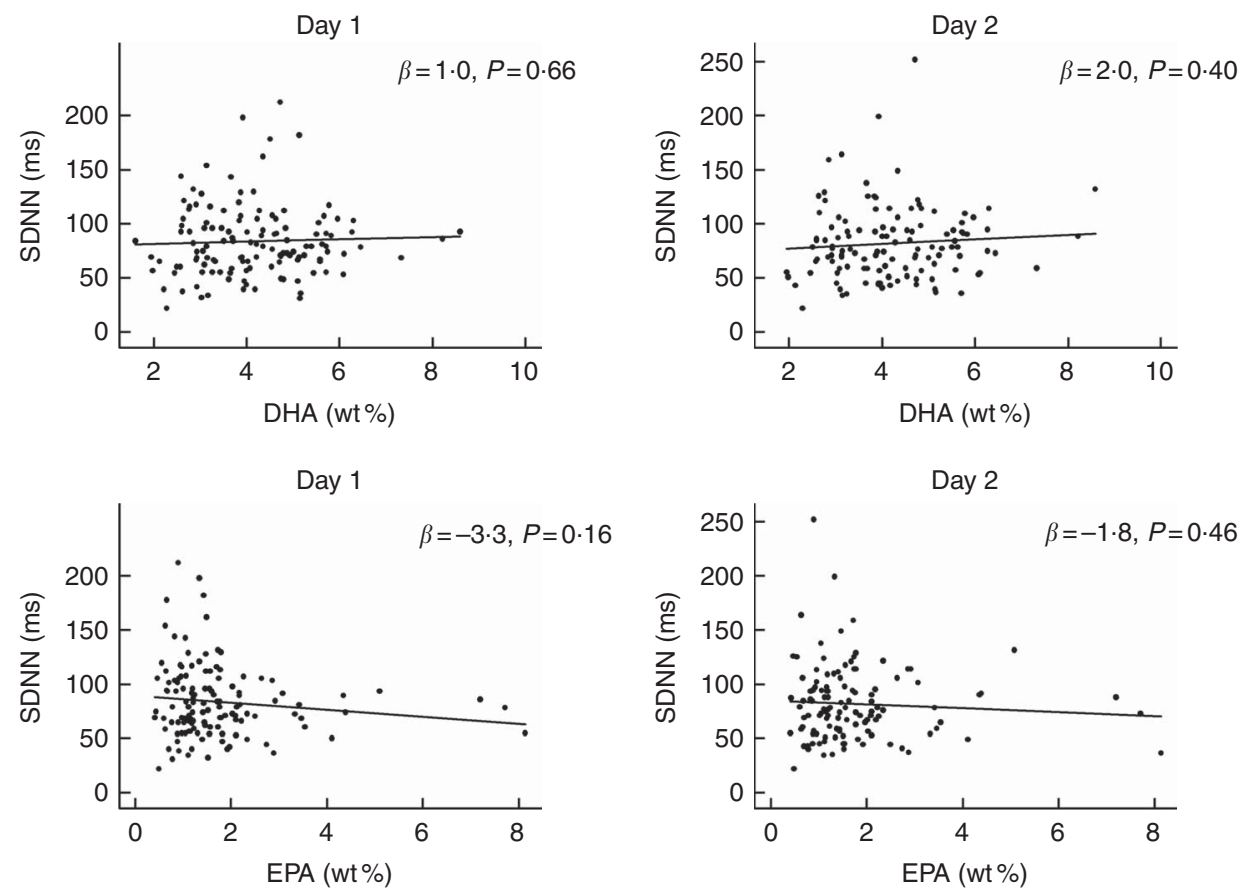

Fig. 3. Associations between DHA or EPA and overall heart rate variability (HRV) (24-h standard deviation of normal intervals (SDNN)) in patients on chronic dialysis. The first column shows results from day 1 and the second column shows results from day 2 . The lines represent the regression lines of the simple linear regression analyses. There was no significant associations between DHA, EPA and SDNN. wt\%, weight percent.

A part of the suggested protective effect of $n$-3 PUFA on SCD may be improvement of autonomic modulation of the heart expressed as an increase in $\mathrm{HRV}^{(20)}$. This has been seen in some studies in high-risk populations with $\mathrm{CHD}^{(35-38)}$, but negative findings have also been reported ${ }^{(39-42)}$. Only two small studies have examined the effects of marine $n$ - 3 PUFA on HRV in patients on chronic dialysis ${ }^{(21,22)}$. In a randomised controlled design ${ }^{(21)}$, twenty-nine HD and PD patients were allocated to $5.2 \mathrm{~g} n$-3 PUFA or placebo for 12 weeks. Only seventeen completed the study that showed no effect between treatment groups, but after supplementation a positive correlation between granulocyte levels of $n$-3 PUFA and 24-h SDNN was noted. In another trial ${ }^{(22)}$, forty-three HD patients with CHD were allocated to $1.7 \mathrm{~g} \mathrm{n}$-3 PUFA daily for 3 months. Only thirty patients were included in the final analysis showing no effects on 24-h time-domain HRV. In the present study, no association was found between plasma phospholipid content of marine $n-3$ PUFA and HRV in patients on chronic dialysis. The reason for this could be a truly absent effect on HRV in this population. These patients have a very high level of co-morbidities and a high degree of autonomic dysfunction, which might result in inability of marine $n$-3 PUFA to exert a beneficial effect on HRV, which also has been suggested in diabetics with overt autonomic neuropathy ${ }^{(43)}$. The use of multiple medication including beta blockers and statins may also have impeded the possibility to show associations between the plasma phospholipid content of $n$-3 PUFA and $\mathrm{HRV}^{(40)}$. ESKD has been associated with a low intake of marine $n$-3 PUFA especially in American cohorts ${ }^{(44,45)}$. In our study the patients had a higher mean plasma phospholipid level of marine $n-3$ PUFA than seen in most of these studies indicating a higher $n-3$ PUFA intake. However, the marine $n$-3 PUFA levels could still be too low to affect autonomic function in this population explaining the negative findings regarding HRV.

The present study is the first to demonstrate that a higher plasma phospholipid content of DHA is associated with a lower presence of non-sustained VT in patients on chronic dialysis. Similar findings with a protective effect of marine $n$-3 PUFA on malignant ventricular arrhythmias have been observed in patients with idiopathic cardiomyopathy ${ }^{(46)}$ and $\mathrm{CHD}^{(42)}$. Chance as a reason for the detected association must be considered due to the cross-sectional design and several endpoints. In line with this, no protective effect of marine $n-3$ PUFA on malignant ventricular arrhythmias was seen in studies on highrisk patients with implantable cardioverter defibrillators ${ }^{(47-50)}$. The finding of a potential protective effect on VT of the plasma phospholipid content of DHA and no observed effect on HRV suggests that an antiarrhythmic effect in this high-risk population mainly is due to a direct effect on the heart and not by improvement of autonomic control. In agreement with our results, it has been suggested that DHA has more potent antiarrhythmic effects than $\mathrm{EPA}^{(17,18)}$. This is also plausible as the myocardial content of DHA is higher than that of $\mathrm{EPA}^{(18)}$.

This study had several strengths. The use of biological measures of plasma phospholipid content of $n$-3 PUFA reduced the risk of information bias. The use of 24-h HRV as an endpoint has the advantage that it is an objective measure with high reproducibility $^{(8)}$. The study population was unselected increasing external validity. Several limitations however also merit consideration. The cross-sectional design makes it impossible to make any conclusions on cause-effect relationships. HRV was only obtainable in a subgroup of 135 patients, but the baseline 
Table 3. Regression coefficients from simple and multivariate linear regression analyses of associations between heart rate variability (HRV) and DHA or EPA content in plasma phospholipids

(Regression coefficients $(\beta)$ and $P$ values)

\begin{tabular}{|c|c|c|c|c|c|c|c|c|}
\hline & \multicolumn{4}{|c|}{$\mathrm{DHA}$} & \multicolumn{4}{|c|}{ EPA } \\
\hline & \multicolumn{2}{|c|}{ Crude $\beta$} & \multicolumn{2}{|c|}{ Adjusted $\beta^{*}$} & \multicolumn{2}{|c|}{ Crude $\beta$} & \multicolumn{2}{|c|}{ Adjusted $\beta^{*}$} \\
\hline & Mean & $P$ & Mean & $P$ & Mean & $P$ & Mean & $P$ \\
\hline \multicolumn{9}{|l|}{ Time-domain } \\
\hline \multicolumn{9}{|l|}{ Day 1} \\
\hline Mean RR (ms) & -0.72 & 0.93 & -11.4 & $0 \cdot 16$ & -1.94 & 0.82 & $-5 \cdot 56$ & 0.48 \\
\hline SDNN (ms) & 1.03 & 0.66 & 0.26 & 0.91 & $-3 \cdot 30$ & 0.16 & -3.35 & 0.14 \\
\hline SDANN (ms) & 1.31 & 0.55 & 0.67 & 0.76 & -2.47 & 0.26 & $-2 \cdot 80$ & 0.19 \\
\hline SDNNi (ms) & -0.37 & 0.70 & -0.70 & 0.47 & -1.83 & 0.06 & -1.42 & 0.13 \\
\hline rMSSD (ms) & 0.14 & 0.84 & -0.65 & 0.65 & -0.75 & 0.28 & -1.06 & 0.13 \\
\hline Triangular Index & 0.14 & 0.83 & 0.03 & 0.97 & $-1 \cdot 13$ & 0.09 & -1.05 & 0.11 \\
\hline \multicolumn{9}{|l|}{ Day 2} \\
\hline Mean RR (ms) & $-3 \cdot 15$ & 0.71 & $-8 \cdot 2$ & 0.33 & $-3 \cdot 68$ & 0.65 & -3.42 & 0.67 \\
\hline SDNN (ms) & 2.05 & 0.40 & 1.75 & 0.51 & $-1 \cdot 78$ & 0.46 & -1.04 & 0.68 \\
\hline SDANN (ms) & 2.39 & 0.31 & 2.08 & 0.42 & $-1 \cdot 17$ & 0.61 & -0.62 & 0.80 \\
\hline SDNNi (ms) & -0.36 & 0.71 & -0.42 & 0.67 & -1.67 & 0.08 & -1.08 & 0.25 \\
\hline rMSSD (ms) & 0.24 & 0.66 & -0.44 & 0.46 & -0.56 & 0.29 & -0.76 & 0.17 \\
\hline Triangular Index & 0.46 & 0.50 & 0.48 & 0.52 & -0.62 & 0.36 & -0.34 & 0.62 \\
\hline \multicolumn{9}{|l|}{ Frequency-domain } \\
\hline \multicolumn{9}{|l|}{ Day 1} \\
\hline $\mathrm{LF}\left(\mathrm{ms}^{2}\right)$ & -4.82 & 0.77 & 1.42 & 0.94 & $-22 \cdot 1$ & 0.19 & $-10 \cdot 6$ & 0.53 \\
\hline $\mathrm{HF}\left(\mathrm{ms}^{2}\right)$ & -0.23 & 0.98 & -0.19 & 0.98 & $-12 \cdot 5$ & 0.12 & $-10 \cdot 4$ & 0.21 \\
\hline LF (nu) & 0.47 & 0.74 & 0.42 & 0.76 & -1.64 & 0.24 & -0.68 & 0.61 \\
\hline $\mathrm{HF}(\mathrm{nu})$ & -0.64 & 0.51 & -0.64 & 0.51 & 0.62 & 0.52 & 0.26 & 0.79 \\
\hline LF:HF ratio & 0.12 & 0.55 & 0.28 & 0.17 & 0.08 & 0.66 & 0.29 & 0.13 \\
\hline \multicolumn{9}{|l|}{ Day 2} \\
\hline $\mathrm{LF}\left(\mathrm{ms}^{2}\right)$ & -7.36 & 0.67 & 2.09 & 0.95 & $-15 \cdot 6$ & 0.36 & $-2 \cdot 78$ & 0.87 \\
\hline $\mathrm{HF}\left(\mathrm{ms}^{2}\right)$ & 0.42 & 0.96 & 0.55 & 0.94 & -9.21 & 0.21 & $-7 \cdot 74$ & 0.32 \\
\hline LF (nu) & 0.20 & 0.89 & 1.01 & 0.49 & $-1 \cdot 81$ & 0.19 & -0.40 & 0.77 \\
\hline $\mathrm{HF}(\mathrm{nu})$ & -0.23 & 0.83 & -0.38 & 0.74 & 1.15 & 0.27 & 0.63 & 0.55 \\
\hline LF:HF ratio & 0.08 & 0.69 & 0.30 & 0.17 & 0.02 & 0.91 & 0.24 & 0.25 \\
\hline
\end{tabular}

Mean RR, 24-h mean value of RR-intervals; SDNN, 24-h standard deviation of normal intervals; SDANN, standard deviation of the mean of RR-intervals in successive 5-min segments; SDNNi, mean of the standard deviation of all normal RR-intervals for all 5-min segments; rMSSD, square root of the mean of the sum of the squares of differences between adjacent intervals; LF, low frequency; nu, normalised units; HF, high frequency.

* Adjustments were done for the following covariates: demography (age; sex; smoking status), co-morbidity (diabetes mellitus; CHD) and use of medication (beta blocker; angiotensin-converting enzyme-inhibitor or angiotensin receptor blocker; statin).

Table 4. Associations between plasma phospholipid marine $n$-3 PUFA levels and ventricular arrhythmias on 48-h Holter monitoring (Medians and interquartile ranges (IQR); numbers and percentages)

\begin{tabular}{|c|c|c|c|c|c|c|c|}
\hline & \multicolumn{2}{|c|}{ 1st $n$-3 PUFA tertile } & \multicolumn{2}{|c|}{ 2nd $n$-3 PUFA tertile } & \multicolumn{2}{|c|}{ 3rd $n-3$ PUFA tertile } & \multirow[b]{2}{*}{$P^{\star}$} \\
\hline & Median & IQR & Median & IQR & Median & IQR & \\
\hline \multicolumn{8}{|l|}{ Number of PVC } \\
\hline DHA tertiles & 57 & 7,716 & 121 & 7,870 & 25 & 4,237 & 0.26 \\
\hline EPA tertiles & 25 & 5,345 & 28 & 7,862 & 60 & 7,327 & 0.78 \\
\hline \multirow[t]{2}{*}{ Total $n$-3 PUFA tertiles } & 28 & 6,322 & 138 & 8,1038 & 42 & 5,237 & 0.27 \\
\hline & $n$ & $\%$ & $n$ & $\%$ & $n$ & $\%$ & \\
\hline \multicolumn{8}{|l|}{ Frequent PVC† } \\
\hline DHA tertiles & 9 & 18 & 10 & 21 & 5 & 9 & 0.24 \\
\hline EPA tertiles & 5 & 10 & 12 & 24 & 7 & 13 & 0.17 \\
\hline Total $n-3$ PUFA tertiles & 6 & 12 & 12 & 23 & 6 & 12 & 0.21 \\
\hline \multicolumn{8}{|l|}{ Ventricular tachycardiał } \\
\hline DHA tertiles & 14 & 28 & 11 & 23 & 5 & 9 & 0.04 \\
\hline EPA tertiles & 9 & 19 & 12 & 24 & 9 & 17 & 0.69 \\
\hline Total $n-3$ PUFA tertiles & 11 & 22 & 13 & 25 & 6 & 12 & 0.20 \\
\hline
\end{tabular}

PVC, premature ventricular complexes.

${ }^{*} \chi^{2}$ Test or Kruskal-Wallis test.

$\dagger>30$ PVCs/h.

$\ddagger \geq 3$ ventricular beats with heart rate $>100$ beats per min. 


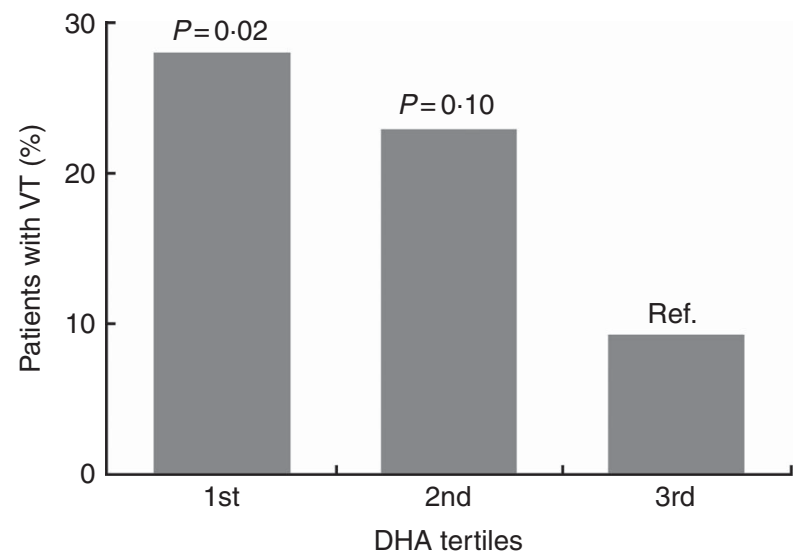

Fig. 4. Patients with episodes of non-sustained ventricular tachycardia (VT) according to level of DHA in plasma phospholipids. $P$ values are for comparisons with the highest tertile as a reference. Comparisons across all three groups $\left(X^{2}\right.$ test) was also significant $(P=0.04)$. The ranges of tertiles are 1st tertile: $1.60-3.45 \mathrm{wt} \%$, 2nd tertile: $3.46-4.64 \mathrm{wt} \%$ and 3 rd tertile: 4.65-8.59 wt\%. wt\%, weight percent.

characteristics and plasma phospholipid content of marine $n$-3 PUFA were comparable suggesting this sample was representative of the entire population. Furthermore, the relatively small sample size increased the risk of failure to detect real associations (type II error). We adjusted for a number of potential confounders, but the heterogeneous nature of chronic dialysis patients increased the risk of residual confounding.

In conclusion, our study did not demonstrate any association between the plasma phospholipid content of marine $n-3$ PUFA and HRV in patients on chronic dialysis. A higher plasma phospholipid content of DHA was however associated with a lower proportion of patients experiencing non-sustained VT suggesting a direct antiarrhythmic effect in this high-risk population. Whether supplementation of $n$ - 3 PUFA has positive effects on autonomic control and ventricular arrhythmias in this patient population remains to be seen and needs to be clarified through adequately powered randomised controlled trials.

\section{Acknowledgements}

The authors thank laboratory technician Charlotte Mose Skov for her assistance in conducting the study and thank laboratory technicians Rikke Bülow Eschen, Anette Andreassen and Birthe Thomsen for analysing plasma phospholipid fatty acids.

This work was supported by the Danish Council for Strategic Research - (AF Study Group), the Arvid Nilsson Foundation, the Danish Kidney Association and the Danish Society of Nephrology. The funders had no role in the design of the study, data collection, data analysis, interpretation, writing the report or decision to submit the report for publication.

The author's contributions were as follows: J. M. R., E. B. S., S. R., J. H. C. designed the study; J. M. R. conducted the study; J. M. R. and S. L.-C. performed the statistical analysis; J. M. R. wrote the first draft and all the authors contributed important intellectual content during manuscript revision and approved the final version of the manuscript.

None of the authors has any conflicts of interests to declare.

\section{References}

1. Herzog CA, Mangrum JM \& Passman R (2008) Sudden cardiac death and dialysis patients. Semin Dial 21, 300-307.

2. National Institute of Health \& National Institutes of Diabetes and Digestive and Kidney Diseases (2016) United States Renal Data System. 2016 USRDS Annual Data Report: Epidemiology of Kidney Disease in the United States. Bethesda, MD: National Institute of Health \& National Institutes of Diabetes and Digestive and Kidney Diseases.

3. Waks JW, Tereshchenko LG \& Parekh RS (2016) Electrocardiographic predictors of mortality and sudden cardiac death in patients with end stage renal disease on hemodialysis. J Electrocardiol 49, 848-854.

4. Hathaway DK, Cashion AK, Milstead EJ, et al. (1998) Autonomic dysregulation in patients awaiting kidney transplantation. Am J Kidney Dis 32, 221-229.

5. La Rovere MT, Bigger JT Jr, Marcus FI, et al. (1998) Baroreflex sensitivity and heart-rate variability in prediction of total cardiac mortality after myocardial infarction. ATRAMI (Autonomic Tone and Reflexes After Myocardial Infarction) Investigators. Lancet 351, 478-484.

6. Kleiger RE, Miller JP, Bigger JT Jr, et al. (1987) Decreased heart rate variability and its association with increased mortality after acute myocardial infarction. Am J Cardiol 59, 256-262.

7. Nishimura M, Tokoro T, Nishida M, et al. (2010) Sympathetic overactivity and sudden cardiac death among hemodialysis patients with left ventricular hypertrophy. Int J Cardiol $\mathbf{1 4 2}$, 80-86.

8. Malik M, Bigger JT, Camm AJ, et al. (1996) Heart rate variability: standards of measurement, physiological interpretation and clinical use. Task Force of the European Society of Cardiology and the North American Society of Pacing and Electrophysiology. Circulation 93, 1043-1065.

9. Oikawa K, Ishihara R, Maeda T, et al. (2009) Prognostic value of heart rate variability in patients with renal failure on hemodialysis. Int J Cardiol 131, 370-377.

10. Hayano J, Takahashi H, Toriyama T, et al. (1999) Prognostic value of heart rate variability during long-term follow-up in chronic haemodialysis patients with end-stage renal disease. Nephrol Dial Transplant 14, 1480-1488.

11. Suzuki M, Hiroshi T, Aoyama T, et al. (2012) Nonlinear measures of heart rate variability and mortality risk in hemodialysis patients. Clin J Am Soc Nephrol 7, 1454-1460.

12. Badarau S, Siriopol D, Drugus D, et al. (2015) Electrocardiogram abnormalities and heart rate variability in predicting mortality and cardiovascular events among hemodialyzed patients. Int Urol Nephrol 47, 1703-1708.

13. Calder PC (2004) n-3 Fatty acids and cardiovascular disease: evidence explained and mechanisms explored. Clin Sci (Lond) 107, 1-11.

14. Albert CM, Campos H, Stampfer MJ, et al. (2002) Blood levels of long-chain $n$-3 fatty acids and the risk of sudden death. $N$ Engl J Med 346, 1113-1118.

15. Marchioli R, Barzi F, Bomba E, et al. (2002) Early protection against sudden death by $n-3$ polyunsaturated fatty acids after myocardial infarction: Time-course analysis of the results of the Gruppo Italiano per lo Studio della Sopravvivenza nell'Infarto Miocardico (GISSI)-Prevenzione. Circulation 105, 1897-1903.

16. Tavazzi L, Maggioni AP, Marchioli R, et al. (2008) Effect of $n-3$ polyunsaturated fatty acids in patients with chronic heart failure (the GISSI-HF trial): a randomised, double-blind, placebo-controlled trial. Lancet 372, 1223-1230.

17. Mozaffarian D, Lemaitre RN, King IB, et al. (2013) Plasma phospholipid long-chain omega-3 fatty acids and total and 
cause-specific mortality in older adults: a cohort study. Ann Intern Med 158, 515-525.

18. McLennan PL \& Abeywardena MY (2005) Membrane basis for fish oil effects on the heart: linking natural hibernators to prevention of human sudden cardiac death. $J$ Membr Biol 206, 85-102.

19. Geelen A, Zock PL, Swenne CA, et al. (2003) Effect of $n$-3 fatty acids on heart rate variability and baroreflex sensitivity in middle-aged subjects. Am Heart J 146, E4.

20. Christensen JH (2003) n-3 Fatty acids and the risk of sudden cardiac death. Emphasis on heart rate variability. Dan Med Bull 50, 347-367.

21. Christensen JH, Aaroe J, Knudsen N, et al. (1998) Heart rate variability and $n-3$ fatty acids in patients with chronic renal failure - a pilot study. Clin Nephrol 49, 102-106.

22. Svensson M, Schmidt EB, Jorgensen KA, et al. (2007) The effect of $n$-3 fatty acids on heart rate variability in patients treated with chronic hemodialysis. J Ren Nutr 17, 243-249.

23. Eide IA, Jenssen T, Hartmann A, et al. (2015) The association between marine $n-3$ polyunsaturated fatty acid levels and survival after renal transplantation. Clin J Am Soc Nephrol 10, 1246-1256.

24. Longenecker JC, Zubaid M, Johny KV, et al. (2009) Association of low heart rate variability with atherosclerotic cardiovascular disease in hemodialysis patients. Med Princ Pract 18, 85-92.

25. da Silva DM, Macedo MC, Lemos LB, et al. (2016) Reliability analysis of the heart autonomic control parameters during hemodialysis sessions. Biomed Tech (Berl) 61, 623-630.

26. Kutner NG, Clow PW, Zhang R, et al. (2002) Association of fish intake and survival in a cohort of incident dialysis patients. Am J Kidney Dis 39, 1018-1024.

27. Hamazaki K, Terashima Y, Itomura M, et al. (2011) Docosahexaenoic acid is an independent predictor of all-cause mortality in hemodialysis patients. Am J Nephrol 33, 105-110.

28. Terashima Y, Hamazaki K, Itomura M, et al. (2014) Inverse association between docosahexaenoic acid and mortality in patients on hemodialysis during over 10 years. Hemodial Int 18, 625-631.

29. Inoue $\mathrm{T}$, Okano K, Tsuruta $\mathrm{Y}$, et al. (2015) Eicosapentaenoic acid (EPA) decreases the all-cause mortality in hemodialysis patients. Intern Med 54, 3133-3137.

30. Friedman AN, Saha C \& Watkins BA (2008) Feasibility study of erythrocyte long-chain omega-3 polyunsaturated fatty acid content and mortality risk in hemodialysis patients. J Ren Nutr 18, 509-512.

31. Friedman AN, Yu Z, Tabbey R, et al. (2013) Inverse relationship between long-chain $n-3$ fatty acids and risk of sudden cardiac death in patients starting hemodialysis. Kidney Int 83, 993-995.

32. Huang X, Stenvinkel P, Qureshi AR, et al. (2012) Essential polyunsaturated fatty acids, inflammation and mortality in dialysis patients. Nephrol Dial Transplant 27, 3615-3620.

33. Svensson M, Schmidt EB, Jorgensen KA, et al. (2006) n-3 Fatty acids as secondary prevention against cardiovascular events in patients who undergo chronic hemodialysis: a randomized, placebo-controlled intervention trial. Clin J Am Soc Nephrol 1, 780-786.

34. Lok CE, Moist L, Hemmelgarn BR, et al. (2012) Effect of fish oil supplementation on graft patency and cardiovascular events among patients with new synthetic arteriovenous hemodialysis grafts: a randomized controlled trial. JAMA 307, 1809-1816.

35. Christensen JH, Skou HA, Fog L, et al. (2001) Marine $n$-3 fatty acids, wine intake, and heart rate variability in patients referred for coronary angiography. Circulation 103, 651-657.
36. Christensen JH, Gustenhoff P, Korup E, et al. (1996) Effect of fish oil on heart rate variability in survivors of myocardial infarction: a double blind randomised controlled trial. $B M J$ 312, 677-678.

37. Villa B, Calabresi L, Chiesa G, et al. (2002) Omega-3 fatty acid ethyl esters increase heart rate variability in patients with coronary disease. Pharmacol Res 45, 475.

38. O'Keefe JH Jr, Abuissa H, Sastre A, et al. (2006) Effects of omega- 3 fatty acids on resting heart rate, heart rate recovery after exercise, and heart rate variability in men with healed myocardial infarctions and depressed ejection fractions. $A m \mathrm{~J}$ Cardiol 97, 1127-1130.

39. Hamaad A, Kaeng Lee W, Lip GY, et al. (2006) Oral omega n3-PUFA therapy (Omacor) has no impact on indices of heart rate variability in stable post myocardial infarction patients. Cardiovasc Drugs Ther 20, 359-364.

40. Erkkilä AT, Schwab US, de Mello VD, et al. (2008) Effects of fatty and lean fish intake on blood pressure in subjects with coronary heart disease using multiple medications. Eur J Nutr 47, 319-328.

41. Carney RM, Freedland KE, Stein PK, et al. (2010) Effect of omega-3 fatty acids on heart rate variability in depressed patients with coronary heart disease. Psychosom Med 72, 748-754.

42. Nodera M, Suzuki H, Yamada S, et al. (2015) Association of Serum $n-3 / n-6$ polyunsaturated fatty acid ratio with T-wave alternans in patients with ischemic heart disease. Int Heart $J$ 56, 613-617.

43. Santini V, Ciampittiello G, Gigli F, et al. (2007) QTc and autonomic neuropathy in diabetes: effects of acute hyperglycaemia and $n-3$ PUFA. Nutr Metab Cardiovasc Dis 17, 712-718

44. Madsen T, Christensen JH, Svensson M, et al. (2011) Marine $n-3$ polyunsaturated fatty acids in patients with end-stage renal failure and in subjects without kidney disease: a comparative study. J Ren Nutr 21, 169-175.

45. Friedman AN, Yu Z, Tabbey R, et al. (2012) Low blood levels of long-chain $n-3$ polyunsaturated fatty acids in US hemodialysis patients: clinical implications. Am J Nephrol 36, 451-458.

46. Nodari S, Metra M, Milesi G, et al. (2009) The role of $n-3$ PUFAs in preventing the arrhythmic risk in patients with idiopathic dilated cardiomyopathy. Cardiovasc Drugs Ther 23, 5-15.

47. Raitt MH, Connor WE, Morris C, et al. (2005) Fish oil supplementation and risk of ventricular tachycardia and ventricular fibrillation in patients with implantable defibrillators: a randomized controlled trial. JAMA 293, 2884-2891.

48. Leaf A, Albert CM, Josephson M, et al. (2005) Prevention of fatal arrhythmias in high-risk subjects by fish oil $n-3$ fatty acid intake. Circulation 112, 2762-2768.

49. Brouwer IA, Zock PL, Camm AJ, et al. (2006) Effect of fish oil on ventricular tachyarrhythmia and death in patients with implantable cardioverter defibrillators: the Study on Omega-3 Fatty Acids and Ventricular Arrhythmia (SOFA) randomized trial. JAMA 295, 2613-2619.

50. Finzi AA, Latini R, Barlera S, et al. (2011) Effects of $n-3$ polyunsaturated fatty acids on malignant ventricular arrhythmias in patients with chronic heart failure and implantable cardioverter-defibrillators: a substudy of the Gruppo Italiano per lo Studio della Sopravvivenza nell'Insufficienza Cardiaca (GISSIHF) trial. Am Heart J 161, 338-343.e1. 